\title{
MULHERES E A MASTECTOMIA: REVISÃO LITERÁRIA
}

\author{
WOMEN AND MASTECTOMY: LITERARY REVIEW
}

\section{Adriana Mary Nunes Costa ${ }^{a^{*}}$, Érica da Rocha Pereira ${ }^{b^{*}}$, Thiago Brasileiro de Vasconcelos ${ }^{c^{*}}$, Maria do Socorro Quintino Farias ${ }^{\mathrm{d**}}$, Liana Rocha Praça ${ }^{\mathrm{e}^{* *}}$, Vasco Pinheiro Diógenes Bastos ${ }^{\mathrm{f**}}$}

\author{
adrianamncosta@gmail.com, bericarocha.rp@hotmail.com, cthiagobvasconcelos@hotmail.com, dqfarias@edu.estacio.br, \\ elianarpraca@yahoo.com.br, fvascodiogenes@yahoo.com.br \\ *Universidade Federal do Ceará. Fortaleza/Ceará \\ ${ }^{* *}$ Centro Universitário Estácio do Ceará
}

\section{RESUMO}

Introdução: $\mathrm{O}$ câncer de mama, dentre as neoplasias malignas, é o segundo tipo de câncer mais frequente e o mais prevalente na classe feminina. Várias são as transformaçóes físicas e emocionais pelas quais passam as mulheres com tal diagnóstico. Objetivo: Revisar a relaçáo física e emocional existente entre as mulheres e a mastectomia. Metodologia: $\mathrm{O}$ método utilizado foi uma revisão literária de bases de dados e revistas especializadas, do período de 2006 a 2012. Foram utilizados os seguintes descritores: "mastectomia", "fisioterapia" e "câncer de mama". Como critérios de inclusão, os estudos deveriam preencher as seguintes condiçóes: apresentar sequelas físicas e emocionais no pós-operatório do procedimento cirúrgico; relacionar a mastectomia com as mulheres. Foram excluídos artigos que não tivessem relação entre a mastectomia e as mulheres. Para a análise dos dados, foram extraídas informaçôes de cada artigo referentes às condiçốes físicas e emocionais no período pós-procedimento cirúrgico, assim como exercícios abordados para melhor recuperação das pacientes, relação entre os procedimentos realizados, alteraçóes físico-emocionais da paciente e a influência da sexualidade após a mastectomia. Resultados: Foram coletados e analisados 25 estudos referentes à mastectomia e às mulheres mastectomizadas. As mulheres mastectomizadas passam por sensaçóes negativas tanto físicas quanto emocionais no período pós-operatório, com destaque para a vida sexual, social e física. As mulheres mastectomizadas comumente apresentam depreciação da imagem corporal, e a aparência física está relacionada com o fato de sentirem o seu corpo incompleto. Conclusão: A mulher mastectomizada necessita do apoio familiar e conjugal e de acompanhamento multiprofissional.

Palavras-chave: mastectomia; câncer de mama; fisioterapia.

\section{ABSTRACT}

Introduction: Breast cancer is the second most common cancer among the malignancies, and the most prevalent in female class. Many are the physical and emotional difficulties for women with this diagnosis. Objective: To review the physical and emotional relationship between women and mastectomy. Methodology: The method used was a literature review of databases and journals in the period of 2006 to 2012, being used the following descriptors: "mastectomy", "therapy" and "breast cancer". For inclusion, studies had to meet the following conditions: physical and emotional sequelae postoperatively the surgical procedure; relate mastectomy with women. It was excluded articles that had no relation between mastectomy and women. For data analysis, it were extracted informations from each article regarding the physical and emotional conditions in the period after surgical procedure, exercise addressed to better recovery of patients, the relationship between the procedures performed, the physical and emotional changes and the influence of patient sexuality after mastectomy. Results: It was collected and analyzed 25 studies related to women who had mastectomies and mastectomy. Women undergo mastectomies negative feelings, both 
physical and emotional in the postoperative period, especially the sexual, social and physical life. Conclusion: Women with mastectomies need family support and marital and multidisciplinary monitoring.

Keywords: mastectomy; breast cancer; physical therapy.

\section{Introdução}

O câncer de mama, dentre as neoplasias malignas, é o segundo tipo de câncer mais frequente, sendo o mais prevalente entre as mulheres ${ }^{1}$. Segundo Forouzanfar et al. ${ }^{2}$, a incidência da doença está aumentando progressivamente, tanto nos países desenvolvidos como nos em desenvolvimento, com uma taxa anual de 3,1\%. Passou de 641.000 casos, em 1980, para 1.643.000 casos, em 2010, sendo responsável por $27 \%$ dos novos casos de câncer diagnosticados em mulheres.

O carcinoma mamário pode ser diagnosticado de diferentes formas, sendo que as mais eficazes de detecção precoce são: a mamografia, que é considerada hoje o instrumento mais efetivo para diagnóstico ${ }^{3}$, o exame clínico, que é um importante método de detecção precoce e que, associado à mamografia, pode aumentar a sensibilidade dos resultados, e, para finalizar, o autoexame das mamas, caracterizado pela facilidade e baixo custo, já que quem o executa é a própria mulher .

Depois de detectado o câncer de mama e constatada a necessidade de intervenção cirúrgica, a equipe médica poderá optar por procedimentos cirúrgicos radicais ou conservadores. O tratamento cirúrgico vai desde a tumorectomia até a mastectomia, associada ou não à retirada dos linfonodos axilares 5 .

Os comprometimentos funcional e emocional relacionados aos procedimentos cirúrgicos mencionados são frequentes no pós-operatório, envolvendo tanto mulheres submetidas ao tratamento conservador como cirurgias radicais. Algumas das principais sequelas envolvidas no pós-operatório são: infecçōes locais, necrose cutânea, retraçôes cicatriciais, disfunçóes respiratórias, linfedema, alteraçóes funcionais, lesōes nervosas, distúrbios da sensibilidade, alteração da amplitude de movimento do ombro e dor ${ }^{6}$.

A dor é frequente no pós-operatório e pode ter início em horas, semanas ou meses, localizando-se normalmente na face anterior do tórax, axila e/ou na metade superior do braço, persistindo por período superior a três meses após a operaçáo. A queixa dolorosa do tipo crônica sucede mais comumente à mastectomia radical com linfadenectomia axilar. A maior frequência de dor não está relacionada ao tipo de procedimento cirúrgico, mas sim à abordagem sobre a axila onde o nervo intercostobraquial, por sua proximidade, pode ser lesado ${ }^{7}$.
A fisioterapia oncológica atua no pós e no pré-operatório de diversas cirurgias, dentre elas a mastectomia, e aborda vários aspectos, desde a preparaçáo do paciente para a cirurgia até a reabilitaçáo funcional no pós-cirúrgico. A paciente que se submete a um programa preventivo no pré-operatório pode diminuir o tempo de internaçấo e retornar mais rapidamente às atividades diárias e ocupacionais. Num contexto mais amplo, um programa fisioterapêutico preventivo auxilia evitando complicaçóes comuns decorrentes do procedimento cirúrgico ${ }^{8,9}$.

Levando-se em consideração o número crescente de mulheres acometidas pela neoplasia mamária, o estudo realizado é de extrema relevância, pois revisou as condiçóes físicas e psicológicas dessas mulheres proporcionando aos profissionais uma melhor análise e, conseguintemente, otimização no tratamento.

Diante disso, o estudo em questáo teve como principal objetivo realizar uma revisão sobre a relação física e emocional existente entre as mulheres e a mastectomia, e, como objetivos específicos, observar a relação da mulher mastectomizada e sua qualidade de vida com a sociedade, com os diferentes procedimentos cirúrgicos utilizados e com a fisioterapia.

\section{Metodologia}

Trata-se de um estudo de revisão bibliográfica, onde realizou-se busca sobre o tema em questáo em artigos publicados em periódicos nacionais e disponibilizados pelo Sistema Bireme (LILACS, SciElo e Base de dados do $\mathrm{CNPq}$ ), sendo todos publicados no período de 2006 a 2012.

Foram utilizados os seguintes descritores: "mastectomia", "fisioterapia", "câncer de mama". Como critérios de inclusão, os estudos deveriam preencher as seguintes condiçôes: apresentar sequelas físicas e emocionais no pós-operatório do procedimento cirúrgico e relacionar a mastectomia com as mulheres.

Foram excluídos artigos que não tivessem relação entre a mastectomia e as mulheres. Para a análise dos dados, foram extraídas informaçôes de cada artigo referentes às condições físicas e emocionais no período pós-procedimento cirúrgico, exercícios abordados para melhor recuperação das pacientes, relação entre os procedimentos realizados, alteraçôes físico-emocionais da paciente e a influência da sexualidade após a mastectomia. 


\section{Resultados e Discussão}

Foram coletados e analisados 25 estudos referentes à mastectomia e às mulheres mastectomizadas. Destes, $44 \%(\mathrm{n}=11)$ eram do tipo transversal, 32\% $(\mathrm{n}=8)$ revisōes literárias, $12 \%(\mathrm{n}=3)$ experimentais, $8 \%(\mathrm{n}=2)$ ensaio clínico e $4 \%(n=1)$ estudo de coorte.

1. Procedimentos cirúrgicos na mastectomia

A discussáo quanto ao melhor método revela na literatura que quanto às consequências físicas no pós-operatório de mastectomia, tanto o procedimento conservador quanto o radical apresentam resultados desde que os conceitos básicos da cirurgia oncológica sejam seguidos de forma rígida. Deve ficar claro que os procedimentos com preservação da mama, quando realizados de forma incorreta, trazem prejuízo para a sobrevida das pacientes com câncer de mama e, portanto, devem ser realizados por profissionais especializados em instituiçóes que fornecem apoio multiespecializado ${ }^{10}$.

A literatura aborda, ainda, uma relação com o tipo de procedimento cirúrgico e a qualidade de vida no pós -operatório. Foi visto que melhor nível socioeconômico e de escolaridade, relação marital estável e cirurgia com conservação mamária estão associados a melhores taxas de qualidade de vida, inclusive a sexual ${ }^{11}$.

Um dos principais problemas da cirurgia mutiladora (radical) é, muitas vezes, a ocorrência de alteraçóes psíquicas, envolvendo morbidades e a interferência na qualidade de vida na mulher, o que levou a uma mudança de conduta cirúrgica ao longo dos anos ${ }^{12}$.

De acordo com Silva e Santos ${ }^{13}$, a reabilitação física e psicossocial, para essas mulheres, não se esgotam com o fim dos procedimentos cirúrgicos, quimio e radioterápicos. Pesquisas abordando o período pós-tratamento são cada vez mais requeridas a fim de conhecer minuciosamente suas especificidades e necessidades. Acrescenta-se a isso, o fato de que os tratamentos antineoplásicos evoluíram muito nas últimas décadas, e estão se tornando extremamente sofisticados e resolutivos, o que resulta em aumento da sobrevida e, consequentemente, em uma preocupação crescente com a qualidade de vida dos pacientes que sobrevivem ao câncer.

\section{Mastectomia e qualidade de vida}

A perda da mama pode levar a consequências negativas na imagem corporal da mulher e, consequentemente, na sua sexualidade. Tal fato é corroborado por estudo realizado por Moniz et al. ${ }^{14}$, no qual as mulheres mastectomizadas apresentam depreciação em relação ao corpo, no geral, e à aparência física, sendo que a maior preocupação dessas mulheres em relação à sua imagem corporal está relacionada com o fato de sentirem o seu corpo incompleto. Algumas das mulheres inquiridas para o estudo mantêm a crença de que o corpo é fundamental na relação entre o homem e a mulher, independentemente da desvalorização que possam ter em relação à sua imagem corporal.

Figura 1: Autoexame das mamas

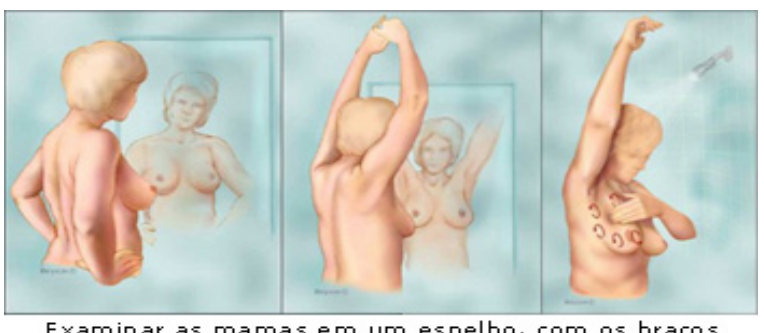

Examinar as mamas em um espelho, com os bracos elevados, observando assimetrias, retrações ou deformidades.

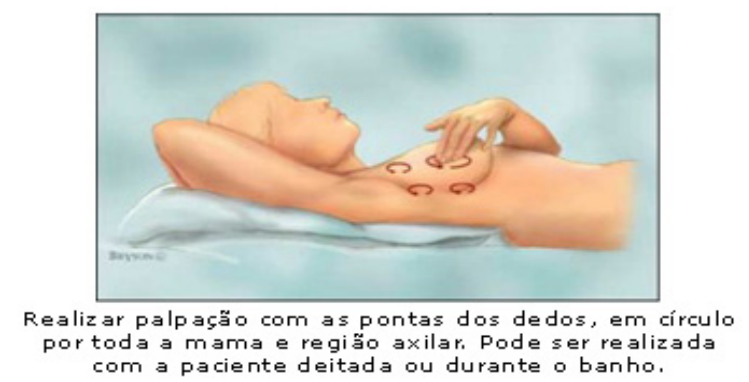

Fonte: www.breastcancer.com

O corpo feminino está fragmentado nos seus símbolos (mama, vagina), naquilo que o diferencia do corpo masculino. Ao mesmo tempo, esses símbolos "cercam" a sua identidade enquanto pessoa na valorização daquilo que a define enquanto mulher, sobretudo na nossa sociedade onde existe a celebração do corpo feminino perfeito e erótico ${ }^{15}$.

A mutilação do corpo foi representada pelas mulheres como perda de pedaços, percepção que têm de seu corpo atual, alterado e estranho, como mostram os relatos encontrados no estudo de Ferreira et al. ${ }^{16}:$ “... Até então você tava perfeitinha e de repente você ta com a metade, então é muito difícil, foi muito ruim, muito difícil!" "Retirar minha mama foi mais difícil do que receber a notícia que eu tava com câncer. Porque sim..., sinto que tirou uma coisa muito importante".

Após estudo com seis mulheres de Vila Velha (Espírito Santo/Brasil), as entrevistadas revelaram que antes do câncer eram mais sociáveis e alegres; porém, após a doença, tornaram-se reservadas, tristes e procuram isolar-se do contato social. Além disso, no que se refere aos relacionamentos amorosos, associam a sexualidade ao aspecto genital e, durante as relaçóes sexuais, sentem-se inibidas e tentam ocultar a mama mutilada ${ }^{17}$. 
Figura 2: Imagem ilustrativa da mastectomia radical
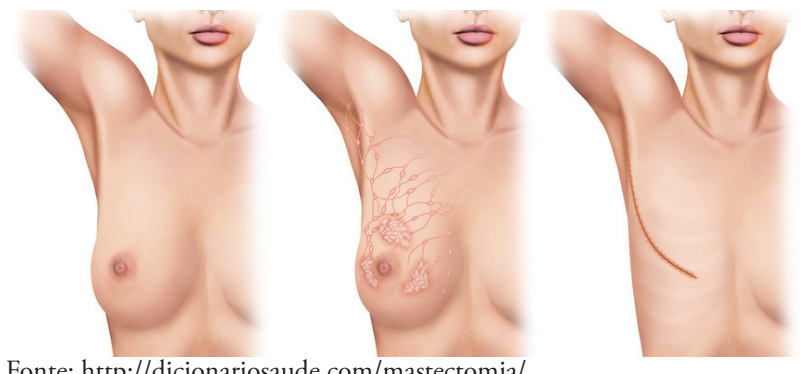

A difícil aceitação e superação da nova condição da sexualidade traz uma relaçáo direta com sentimentos desconhecidos. A depressáo ou o aparecimento de sentimentos negativos na mulher mastectomizada torna-se mais frequente no pós-mastectomia ${ }^{18}$.

Em um estudo feito com 13 mulheres pós mastectomia em hospital de referência em Teresina, cidade do Piauí, Brasil, foi relatado que as entrevistadas apresentavam sentimentos de medo, acanhamento, estranheza, tristeza, espanto e desânimo, ou seja, de desolação em relação à situação de mutilação a que foram submetidas, sendo que o choque existente em relação à percepçáo física é mais evidente após a visualização do resultado da mastectomia ${ }^{18}$.

A literatura mostra que a mastectomia pode trazer intercorrências às mulheres que a realizam, dentre as quais podemos destacar a síndrome dolorosa pós mastectomia e o desenvolvimento de linfedemas, que ocorre em alguns casos, podendo afetar diretamente atividades da vida diária e resultando em uma deficiência na qualidade de vida ${ }^{19}$.

A frequência da síndrome dolorosa pós-mastectomia é alta, variando entre 20 e $50 \%$. O medo de movimentar o membro e a inatividade no pós-operatório leva ao comprometimento gradual da força muscular e da flexibilidade, além do prejuízo na amplitude de movimento, o que predispóe ao aparecimento da dor. O sintoma dor merece atenção por estar diretamente relacionado ao bem estar físico e emocional, ao desempenho e realização das atividades da vida diária, bem como à qualidade de vida ${ }^{19-21}$.

A manifestação de linfedema, outra intercorrência da mastectomia, afeta a qualidade de vida das mulheres que o desenvolvem, pois as mesmas têm que se adequar à nova situaçáo e desenvolver uma série de cuidados para diminuir a probabilidade de desenvolvimento do edema linfático do membro superior homolateral ao procedimento cirúrgico ${ }^{22}$.

Segundo Panobianco, Mamede e Almeida ${ }^{22}$, pelo fato de conviverem com o linfedema, as mulheres relataram, sobre si mesmas, sentimentos como medo e preocupação, além de privarem-se de coisas que lhes davam prazer. Assim, passaram a planejar e organizar suas açóes em função daquelas coisas representadas sobre o significado do linfedema, ou seja, preocupando-se, exercendo um autocuidado e privando-se.

Pode-se imaginar que, após o procedimento cirúrgico, as mulheres mastectomizadas não precisaráo submeter-se a nenhum outro tratamento. No entanto, dá-se início à quimioterapia, além do que muitas mulheres relatam efeitos colaterais do tratamento, como, por exemplo, enjoos e mal-estar físico ${ }^{23}$.

\section{Mastectomia e sociedade}

O câncer de mama desperta diversas reaçóes e sentimentos na sociedade, pois, culturalmente, é visto como uma doença que pode levar à morte. Isso implica o afastamento das pessoas quando o diagnóstico de câncer de mama é recebido, muitas vezes pela dificuldade da sociedade em lidar com uma situação de doença que ameaça a vida ${ }^{24,25}$.

As relaçóes familiares inicialmente podem apresentar-se abaladas, justamente pelo medo da letalidade frequente nessa patologia. A desestruturação familiar pode acontecer em alguns casos, e o apoio dado à mulher pelo cônjuge e familiares torna-se imprescindível ${ }^{26}$.

A convivência com uma mulher com câncer de mama pode ser considerada como fator de sobrecarga física e emocional para a família, que repercute sobre a saúde dos seus membros. Os envolvidos vivenciam momentos de extrema ansiedade, culpa, medo e raiva, tornando o câncer potencialmente mais desestruturador para as famílias do que para os pacientes. Essa desestruturação diz respeito a repercussões físicas, psíquicas e sociais em longo prazo para os familiares ${ }^{26}$.

A dedicação por parte do(a) companheiro(a) é de extrema importância para a mulher, principalmente nas etapas iniciais da doença e mesmo após a mastectomia. Isso porque, com a doença, a mulher sente a necessidade de ser aceita, de afeição, compreensão e carinho. $\mathrm{Na}$ falta desses sentimentos, o relacionamento sexual fica, na compreensão delas, totalmente desestimulante ${ }^{27}$.

\section{Mastectomia e Fisioterapia}

Pesquisas mostram que o tratamento fisioterápico é imprescindível para qualquer indivíduo cuja atividade diária esteja comprometida. Nos processos de doença, contribui para a redução de quadros dolorosos e evita possíveis complicaçóes após cirurgias ou longos períodos de imobilizaçôes, além de despertar para o tratamento oncológico precoce que vem desempenhando um importante papel na prevenção e minimização dos efeitos adversos do tratamento do câncer de mama. 
A fisioterapia reduz os riscos de complicaçóes e pode restaurar a integridade cinético-funcional de órgãos e sistemas $^{8}$.

Alguns autores ${ }^{28,29}$ destacam, como forma de tratamento no pós-operatório imediato, exercícios de membro superior e cervical, posturais e respiratórios, pois a mulher mastectomizada pode apresentar limitaçóes na articulação do ombro, especialmente nos de abdução e flexão anterior, como também rotação externa associada à abdução.

O fisioterapeuta deve prezar pela prevençáo na sua atuação, porém, antecipar possíveis complicações é de responsabilidade de todos os profissionais envolvidos na equipe multidisciplinar, sendo necessária a implementação de medidas preventivas e aconselhamento dos pacientes e familiares para evitar sofrimentos desnecessário ${ }^{30}$.

Um estudo clínico de corte prospectivo e randomizado realizado no Serviço de Fisioterapia do Centro de Atenção Integral à Saúde da Mulher (CAISM), da Universidade Estadual de Campinas (UNICAMP), com 59 pacientes submetidas ao procedimento cirúrgico radical e conservador, mostra que o acompanhamento da fisioterapia precocemente, com a realização dos exercícios ativos e de alongamento, com amplitude livre desde o primeiro dia de pós-operatório, permite boa recuperação da capacidade funcional do ombro homolateral ao procedimento sem aumento de seroma ou deiscência ${ }^{31}$.

\section{Considerações Finais}

O câncer de mama é uma patologia de incidência crescente entre as mulheres. Os procedimentos cirúrgicos empregados para o tratamento de tal doença têm apresentado resultados positivos, e sáo selecionados da forma mais correta em relação ao estadiamento do câncer; porém, o pós-operatório pode apresentar uma gama de intercorrências físicas e psicológicas.

O acompanhamento da mulher com câncer de mama por uma equipe multidisciplinar é de fundamental importância para uma melhor evolução na reinserção da mastectomizada no ambiente social, profissional e pessoal.

O apoio da família e, principalmente do cônjuge, é de fundamental importância para que a mulher perceba que pode ter vida sexual normal.

A fisioterapia tem tido um papel fundamental na manutenção cinético-funcional da cintura escapular e restabelecimento dos movimentos de membros superiores.

\section{Referências}

1. Miranda CMNR, Santos CJJ, Maranhão CPM, Farias LPG, Padilha IG, Andrade ACM et al. A tomografia computadorizada multislice é ferramenta importante para o estadiamento e seguimento do câncer de mama? Radiol Bras. 2012;45(2):105-12.

2. Forouzanfar MH, Foreman KJ, Delossantos AM, Lozano R, Lopez AD, Murray CJL et al. Breast and cervical cancer in 187 countries between 1980 and 2010: a systematic analysis. The Lancet. 2011;378(9824):1461-84.

3. Matos DL, Costa MFL. Prevalência e fatores associados à realização da mamografia na faixa etária de 50-69 anos: um estudo baseado na pesquisa nacional por amostra de domicílios (2003). Cad Saúde Pública. 2007;23(7):1665-73.

4. Costa JSD, Olinto MTA, Bassani D, Marchionatti CRE, Bairros SF, Oliveira MLP et al. Desigualdades na realização do exame clínico de mama em São Leopoldo, Rio Grande do Sul, Brasil. Cadernos de Saúde Pública. 2007;23(7):1603-12.

5. Rett MT, Mesquita PJ, Mendonça ARC, Moura DP, DeSantana JM. A cinesioterapia reduz a dor no membro superior de mulheres submetidas à mastectomia ou quadrantectomia. Rev Dor São Paulo. 2012;13(3):201-7.

6. Bregagnol RK, Dias AS. Alteraçóes funcionais em mulheres submetidas à cirurgia de mama com linfadenectomia axilar total. Rev Bras Cancerol. 2010;56(1):25-33.

7. Menezes CT, Valença MM. Síndrome dolorosa pós mastectomia: A magnitude do problema. Rev Bras Anestesiol. 2009;59(3):358-65.

8. Faria L. As práticas do cuidar na oncologia: a experiência da fisioterapia em pacientes com câncer de mama. Hist cienc saúde-Manguinhos. 2010;17(1):69-87.

9. Loureiro LP, Vasconcelos TB, Martins MEV, Pinheiro CPO, Macena RHM, Bastos VPD. Incidência de complicaçóes pulmonares em mulheres mastectomizadas no pós-operatório imediato. Ensaios e Ciência. 2012;16(1):95-107.

10. Tiezzi DG. Cirurgia conservadora no câncer de mama. Rev Bras Ginecol Obstet. 2007; 29(8):428-34.

11. Veiga DF, Campos FSM, Ribeiro LM, Juliano Y, Neto MS, Ferreira LM et al. Mastectomia versus tratamento cirúrgico conservador: impacto na qualidade de vida de mulheres com câncer mamário. Rev Bras Saude Mater Infant. 2010;10(1):51-7.

12. Maieski VM, Sarquis LMM. Mulheres com câncer de mama em quimioterapia e sua influência sobre o trabalho. Cogitare Enferm. 2007;12(3):346-52.

13. Silva G, Santos MA. "Será que não vai acabar nunca?": perscrutando o universo do pós-tratamento do câncer de mama. Texto contexto - enferm. 2008;17(3):561-68.

14. Moniz PAF, Fernandes AM, Oliveira L. Implicaçóes da mastectomia na sexualidade e imagem corporal da mulher e resposta da enfermagem perioperatória. Rev Enf Ref.2011;3(5):163-71.

15. Aureliano WA. “... e Deus criou a mulher”: reconstruindo o corpo feminino na experiência do câncer de mama. Rev Estud Fem. 2009; 17(1):49-70.

16. Ferreira DB, Farago PM, Reis PED, Funghetto SS. Nossa vida após o câncer de mama: percepçôes e repercussões sob 
o olhar do casal. Rev bras enf. 2011;64(3):536-44.

17. Duarte TP, Andrade NA. Enfrentando a mastectomia: análise dos relatos de mulheres mastectomizadas sobre questôes ligadas à sexualidade. Est Psicol. 2008;8(1):155-63.

18. Moura FMJSP, Silva MG, Oliveira SC, Moura LJSP. Os sentimentos das mulheres pós-mastectomizadas. Esc Anna Nery. 2010;14(3):477-84.

19. Couceiro TCM, Menezes TC, Valença MM. Síndrome dolorosa pós-mastectomia. A magnitude do problema. Rev Bras Anestesiol. 2009;59(3):358-65.Lahoz MA, Nyssen SM, Correia GN, Garcia APU, Driussa P. Capacidade funcional e qualidade de vida em mulheres pós-mastectomia. Rev Bras Cancerol. 2010;56(4):423-30.

20. Costa AIS, Chaves MD. Dor em pacientes oncológicos sob tratamento quimioterápico. Rev Dor. 2012;13(1):45-9.

21. Panobianco MS, Mamede MV, Almeida AM. Experiência de mulheres com linfedema pós-mastectomia: significado do sofrimento vivido. Psicol Estud. 2008;13(4):807-16.

22. Verde SMML, Pedro BMOS, Damasceno NRT, Mourão Neto M. Aversão alimentar adquirida e qualidade de vida em mulheres com neoplasia mamária. Rev Nutr. 2009;22(6):795-807.

23. Pereira SG, Rosenhein DP, Bulhosa MS, Lunardi VL, Filho WDL. Vivências de cuidados da mulher mastectomizada: uma pesquisa bibliográfica. Rev Bras Enf. 2006;59(6):791-5.
24. Bandeira D, Sand ICPVD, Cabral FB, Flores JS, Maron LC, Santos M et al. Repercussóes da mastectomia nas esferas pessoal, social e familiar para a mulher mastectomizada: Uma revisão. Rev Contexto e Saúde. 2011;10(20):473-82.

25. Tavares JSC, Trad LAB. Famílias de mulheres com câncer de mama: desafios associados com o cuidado e os fatores de enfrentamento. Interface (Botucatu). 2009;13(29):395-408.

26. Silva TBC, Santos MCL, Almeida AM, Fernandes AFC. Percepção dos cônjuges de mulheres mastectomizadas com relação à convivência pós-cirurgia. Rev esc enferm. 2010;44(1):113-19.

27. Camargo MC, Marx AG. Reabilitação física no câncer de mama. São Paulo: Roca; 2000.

28. Bergmann A, Ribeiro MJP, Pedrosa E, Nogueira EA, Oliveira ACG. Fisioterapia em mastologia oncológica: rotinas do Hospital do Câncer III/INCA. Rev Cancerol. 2006;52(1):97-109.

29. Marcucci FCI. O papel da fisioterapia nos cuidados paliativos a pacientes com câncer. Rev Bras Cancerol. 2005;51(1):67-77.

30. Silva MPP, Derchain SFM, Rezende L, César Cabello C, Martinez EZ. Movimento do ombro após cirurgia por carcinoma invasor da mama: estudo randomizado prospectivo controlado de exercícios livres versus limitados a $90^{\circ}$ no pós -operatório. Rev Bras Ginecol Obstet. 2010;26(2):125-30. 